\title{
JORDAN FORMS OF REAL AND COMPLEX MATRICES UNDER RANK ONE PERTURBATIONS
}

\author{
Christian Mehl, Volker Mehrmann, André C. M. Ran and Leiba \\ RODMAN
}

Abstract. New perturbation results for the behavior of eigenvalues and Jordan forms of real and complex matrices under generic rank one perturbations are discussed. Several results that are available in the complex case are proved as well for the real case and the assumptions on the genericity are weakened. Rank one perturbations that lead to maximal algebraic multiplicities of the "new" eigenvalues are also discussed.

Mathematics subject classification (2010): 15A18, 15A57, 47A55, 93C73.

Keywords and phrases: Eigenvalues, generic perturbation, rank one perturbation, Jordan canonical form.

\section{REFERENCES}

[1] A. G. AKRItAS, Sylvester's Forgotten Form of the Resultant, Fib. Quart. 31 (1993), 325-332.

[2] R. Alam, S. Bora, M. Karow, V. Mehrmann, and J. Moro, Perturbation theory for Hamiltonian matrices and the distance to bounded-realness, SIAM J. Matrix Anal. Appl., to appear, 2011.

[3] C. P. Coelho, J. R. PhilliPs, AND L. M. Silveira, Robust rational function approximation algorithm for model generation, In Proceedings of the 36th DAC, pages 207-212, New Orleans, Louisiana, USA, 1999.

[4] R. W. Freund And F. JARRE, An extension of the positive real lemma to descriptor systems, Optimization methods and software, 19 (2004), 69-87.

[5] F. R. Gantmacher, Theory of Matrices, volume 1, Chelsea, New York, 1959.

[6] I. GOHBERG, M. A. KAASHOEK, AND L. LERER, The resultant for regular matrix polynomials and quasi commutativity, Ind. Univ. Math. J. 57 (2008), 2793-2813.

[7] I. Gohberg, P. Lancaster, And L. Rodman, Matrix Polynomials, Academic Press, New YorkLondon, 1982; republication, SIAM, 2009.

[8] S. GRIVET-Talocia, Passivity enforcement via perturbation of Hamiltonian matrices, IEEE Trans. Circuits Systems, 51 (2004), 1755-1769.

[9] J. W. Helton AND L. Rodman, Vandermonde and resultant matrices: an abstract approach, Math. Syst. Theory 20 (1987), 169-182.

[10] L. HöRMANDER AND A. Melin, A remark on perturbations of compact operators, Math. Scand. 75 (1994), 255-262.

[11] M. KRUPNIK, Changing the spectrum of an operator by perturbation, Linear Algebra Appl. 167 (1992), 113-118.

[12] M. A. LAIDACKeR, Another Theorem Relating Sylvester's Matrix and the Greatest Common Divisor, Math. Magazine 42 (1969), 126-128.

[13] P. Lancaster and L. Rodman, The Algebraic Riccati Equation, Oxford University Press, Oxford, 1995.

[14] P. Lancaster and M. Tismenetsky, The Theory of Matrices, 2nd edition, Academic Press, Orlando, FL, 1985.

[15] C. Mehl, V. Mehrmann, A. C. M. Ran, and L. Rodman, Eigenvalue perturbation theory of classes of structured matrices under generic structured rank one perturbations, Linear Algebra and its Applications 435 (2011), 687-716. 
[16] C. Mehl, V. Mehrmann, A. C. M. Ran, And L. Rodman, Perturbation theory of selfadjoint matrices and sign characteristics under generic structured rank one perturbations, Linear Algebra and its Applications, DOI: http://dx.doi.org/10.1016/j.laa.2010.04.008, 2011.

[17] V. Mehrmann, The Autonomous Linear Quadratic Control Problem, Theory and Numerical Solution, Number 163 in Lecture Notes in Control and Information Sciences, Springer-Verlag, Heidelberg, 1991.

[18] J. Moro And F. Dopico, Low rank perturbation of Jordan structure, SIAM J. Matrix Anal. Appl. 25 (2003), 495-506.

[19] D. SARASWAT, R. ACHAR, AND M. NAKHIA, On passivity check and compensation of macromodels from tabulated data, In 7th IEEE Workshop on Signal Propagation on Interconnects, pages 25-28, Siena, Italy, 2003.

[20] S. V. SAVCHENKo, Typical changes in spectral properties under perturbations by a rank-one operator, Mat. Zametki 74 (2003), 590-602 (Russian). Translation in Mathematical Notes 74 (2003), $557-568$.

[21] S. V. SAVCHENKO, On the change in the spectral properties of a matrix under a perturbation of a sufficiently low rank, Funktsional. Anal. i Prilozhen 38 (2004), 85-88 (Russian). Translation in Funct. Anal. Appl. 38 (2004), 69-71.

[22] C. Schröder AND T. STYKel, Passivation of LTI systems, Technical Report 368, DFG Research Center Matheon, Technische Universität Berlin, Straße des 17. Juni 136, 10623 Berlin, Germany, 2007.

[23] H. Shapiro, The Weyr characteristic, Amer. Math. Monthly 106 (1999), 919-929.

[24] F. DE TERÁN AND F. M. DoPICO, Low rank perturbation of regular matrix polynomials Linear Algebra Appl., 430 (2009), 579-586. 